S109 TARGETING THE PROSTACYCLIN PATHWAY IN THE TREATMENT OF CONNECTIVE TISSUE DISEASE ASSOCIATED PULMONARY ARTERIAL HYPERTENSION (PAH): INSIGHTS FROM THE RANDOMISED CONTROLLED GRIPHON TRIAL WITH SELEXIPAG

${ }^{1} \mathrm{G}$ Coghlan, ${ }^{2} \mathrm{~S}$ Gaine, ${ }^{3} \mathrm{R}$ Channick, ${ }^{4} \mathrm{~L}$ Di Scala, ${ }^{5} \mathrm{~N}$ Galiè, ${ }^{6,7} \mathrm{HA}$ Ghofrani, ${ }^{8} \mathrm{MM}$ Hoeper, ${ }^{9} \mathrm{I}$ Lang, ${ }^{10} \mathrm{~V}$ McLaughlin, ${ }^{4} \mathrm{R}$ Preiss, ${ }^{11} \mathrm{~L}$ Rubin, ${ }^{12} \mathrm{G}$ Simonneau, ${ }^{12} \mathrm{O}$ Sitbon, ${ }^{13} \mathrm{VF}$ Tapson, ${ }^{14} \mathrm{~K}$ Chin. ${ }^{1}$ National Pulmonary Hypertension Service, Royal Free Hospital, National Health Service Foundation Trust, London, UK; ${ }^{2}$ National Pulmonary Hypertension Unit, Mater Misericordiae University Hospital, Dublin, Ireland; ${ }^{3}$ Massachusetts General Hospital, Boston, MA, USA; ${ }^{4}$ Actelion Pharmaceuticals Ltd, Allschwil, Switzerland; ${ }^{5}$ Istituto di Malattie dell'Apparato Cardiovascolare, University of Bologna, Bologna, Italy; ${ }^{6}$ University of Giessen and Marburg Lung Centre (UGMLC), member of the German Centre of Lung Research (DZL), Giessen, Germany; ' Department of Medicine, Imperial College London, London, UK; ${ }^{8}$ Department of Respiratory Medicine, Hannover Medical School and German Centre of Lung Research, Hannover, Germany; ${ }^{9}$ Medical University of Vienna, Department of Internal Medicine II, Division of Cardiology, Allgemeines Krankenhaus, Vienna, Austria; ${ }^{10}$ University of Michigan Health System Division of Cardiovascular Medicine, Ann Arbor, MI, USA; ${ }^{11}$ Division of Pulmonary and Critical Care Medicine, University of California, San Diego, CA, USA; ${ }^{12}$ Hôpital Universitaire de Bicêtre, Université Paris-Sud, Le Kremlin Bicêtre, France; ${ }^{13}$ Cedars-Sinai Medical Centre, Los Angeles, CA, USA; ${ }^{14}$ UT Southwestern Medical Centre, Dallas, TX, USA

\subsection{6/thoraxjnl-2016-209333.115}

Rationale Despite available therapies, patients with connective tissue disease-associated PAH (PAH-CTD) have a poor prognosis. The global phase III GRIPHON study (NCT01106014) enrolled 1,156 patients including 334 with PAH-CTD. Compared with placebo, selexipag reduced the risk of the primary composite outcome of morbidity/mortality up to end of treatment by $41 \%$ (hazard ratio [HR] 0.59; 99\% CI: 0.37-0.96) among patients with PAH-CTD. We examined the effect of selexipag vs placebo in the PAH-CTD subgroups: PAH associated with systemic sclerosis (PAH-SSc), systemic lupus erythematous (PAH-SLE) and mixed CTD (PAH-MCTD).

Methods Patients (18-75 years) were randomised 1:1 to placebo or selexipag. HRs $(95 \% \mathrm{CI})$ were calculated using Cox regression models to determine the effect of selexipag vs placebo on morbidity/mortality.
Results Of the 334 patients enrolled with PAH-CTD, 170 had PAH-SSc, 82 PAH-SLE, and 47 PAH-MCTD; CTD sub-classification was not reported in 35 patients. Across the subgroups, the majority of patients were female (84-99\%) and were receiving an endothelin receptor antagonist, a phosphodiesterase type-5 inhibitor or both at baseline (73-83\%). In the PAH-SSc, PAH-SLE and PAH-MCTD subgroups, the mean (SD) age was 60.0 (10.6), 39.0 (11.3) and 48.0 (14.7) years, respectively, and 65\%, 33\%, and $45 \%$ were in WHO functional class III, respectively. Selexipag reduced the risk of morbidity/mortality events by $44 \%$ (HR 0.56 ; 95\% CI: 0.34-0.91) in PAH-SSc, 34\% (HR 0.66; 95\% CI: $0.30-$ 1.48 ) in PAH-SLE, and 53\% (HR 0.47; 95\% CI: 0.15-1.48) in PAH-MCTD (Figure). The treatment effect was consistent across the subgroups (interaction test indicated no heterogeneity; $\mathrm{p}=0.6737)$. By the end of study, $22 \mathrm{PAH}-\mathrm{SSc}, 7$ PAH-SLE and 3 PAH-MCTD patients in the placebo, and 17 PAH-SSc, 4 PAHSLE, 8 PAH-MCTD patients in the selexipag group had died. Common prostacyclin-associated side effects observed with selexipag in PAH-CTD patients generally occurred at a similar incidence to PAH-non-CTD patients and within the PAH-CTD subgroups.

Conclusion GRIPHON included the largest randomised cohort of patients with PAH-CTD to date. The treatment effect of selexipag on time to first morbidity/mortality event was consistent across the subgroups, suggesting that selexipag is an effective therapeutic option in these difficult-to-treat patients.

Please refer to page A270 for declarations of interest in relation to abstract S109.

\section{S110 2-D SEGMENTAL LONGITUDINAL STRAIN RATES CORRELATE WITH PROGNOSTIC INDICATORS IN IDIOPATHIC PULMONARY ARTERIAL HYPERTENSION}

${ }^{1} \mathrm{TM}$ Crowe, ${ }^{2} \mathrm{P}$ Sonecki, ${ }^{1} \mathrm{~A}$ Mackenzie, ${ }^{1} \mathrm{G}$ Jayasekera, ${ }^{1} \mathrm{AC}$ Church, ${ }^{1} \mathrm{MK}$ Johnson, ${ }^{1}$ AJ Peacock. 'Scottish Pulmonary Vascular Unit, Glasgow, UK; ${ }^{2}$ Golden Jubilee National Hospital, Glasgow, UK

10.1136/thoraxjnl-2016-209333.116

\begin{tabular}{|c|c|c|c|c|}
\hline Subgroup & $\begin{array}{l}\text { Selexipag } \\
\text { no. of patie }\end{array}$ & $\begin{array}{l}\text { Placebo } \\
\text { 10. of events }\end{array}$ & Hazard ratio and $95 \% \mathrm{Cl}$ & $\begin{array}{l}\mathrm{p} \text {-value for } \\
\text { interaction }\end{array}$ \\
\hline Overall FAS & $574 / 155$ & $582 / 242$ & & \\
\hline Overall CTD & $167 / 48$ & $167 / 73$ & $\mapsto \mid-1$ & \\
\hline CTD population & & & & 0.6737 \\
\hline $\mathrm{PAH}-\mathrm{SSc}$ & $77 / 25$ & $93 / 46$ & $\longmapsto-1$ & \\
\hline PAH-SLE & $45 / 11$ & $37 / 13$ & -1 & \\
\hline PAH-MCTD & $29 / 5$ & $18 / 7$ & -1 & \\
\hline
\end{tabular}

'Other' CTD ( $n=35)$ not shown.

$\mathrm{Cl}$, confidence interval; CTD, connective tissue disease; FAS, full analysis set; MCTD, mixed connective tissue disease; $\mathrm{PAH}$, pulmonary arterial hypertension; SLE, systemic lupus erythematosus; SSc, systemic sclerosis. 
Background/objectives Speckle tracking echocardiography (STE) has added to the assessment of right ventricular function by providing off-line measurements of strain and strain rate. Worsening right ventricular function is associated with poorer outcomes in idiopathic pulmonary arterial hypertension (IPAH). Global right ventricular free wall (RVFW) longitudinal strain and strain rate have been reported as markers of prognosis. Differences in degree of regional RVFW deformation will affect the global RVFW analysis. We assessed whether RVFW segmental analysis using STE identified specific regional associations with proven prognostic markers.

Method Using our database, we identified newly diagnosed cases of IPAH between January 2012 and May 2016. 25 cases had echocardiograms accessible for retrospective analysis. Using 2DSTE software (EchoPAC, GE Healthcare), we measured longitudinal strain and strain rate of the RVFW basal, mid and apical segments. Data on established prognostic markers, NT-proBNP, cardiac index (CI), WHO functional class (FC), six-minute walk distance (6MWD) and mixed venous saturations (SvO2) were retrieved from the patient database. All data was anonymised for analysis.

Results Basal peak systolic longitudinal strain rate (PSLSR) of the RVFW correlated very strongly with NT-proBNP ( $\mathrm{r}=0.82$, $\mathrm{p}<0.001)$ and moderately with CI: $(\mathrm{r}=-0.53, \mathrm{p}<0.007)$, WHO FC $(\mathrm{r}=0.52, \mathrm{p}<0.008)$ and SvO2 $(\mathrm{r}=-0.50$, $\mathrm{p}<0.02$ ). Basal late diastolic longitudinal strain rate (LDLSR) correlated strongly with $\mathrm{SvO} 2(\mathrm{r}=0.61, \mathrm{p}<0.002)$, and moderately with NT-proBNP ( $\mathrm{r}=-0.58, \mathrm{p}<0.005)$, CI: $(\mathrm{r}=0.57$, $\mathrm{p}<0.004), 6 \mathrm{MWD}(\mathrm{r}=0.5, \mathrm{p}<0.02)$, and WHO FC $(\mathrm{r}=-0.49, \mathrm{p}<0.02)$. Mid PSLSR and LDLSR moderately correlated with $\mathrm{SvO} 2(\mathrm{r}=-0.53$ and 0.56$)$, NT-proBNP $(\mathrm{r}=0.5$ and -0.5$)$ and CI ( $r=-0.48$ and 0.42$)$, (all p < 0.04). The apical segment showed no significant correlations. Peak systolic longitudinal strain demonstrated only one significant segmental correlation between the mid-segment and NT-proBNP $(r=0.44$, $\mathrm{p}<0.05)$.

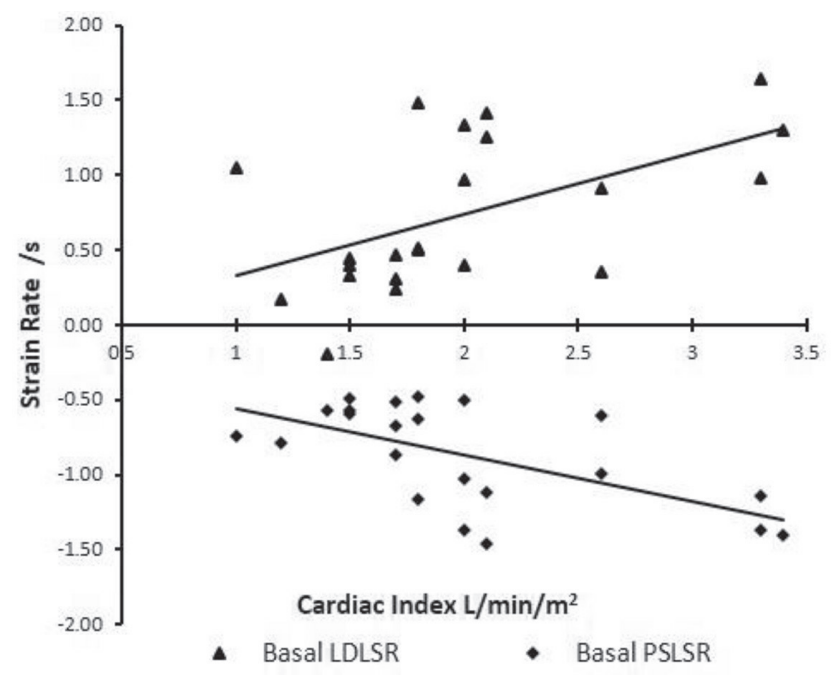

Abstract S110 Figure 1 Basal Late Diastolic and Peak Systolic Longitudinal Strain Rates versu Cardiac Index

Conclusion We have demonstrated that analysing RVFW longitudinal segments provides significant regional correlations with known prognostic markers in an IPAH population group. We have shown the significant advantage of longitudinal strain rate over strain in its strength and consistency of correlation with these markers. We have acknowledged basal and mid segments as significant regions of interest. This study shows that further exploration of right ventricular function in pulmonary hypertension by RVFW segmental analysis is indicated.

\section{S111 DIFFERENCES IN CHARACTERISTICS AND OUTCOMES IN SYSTEMIC SCLEROSIS-ASSOCIATED AND IDIOPATHIC PULMONARY ARTERIAL HYPERTENSION}

S Ramjug, N Hussain, J Hurdman, C Billings, CA Elliot, DG Kiely, I Sabroe, S Rajaram, AJ Swift, R Condliffe. Royal Hallamshire Hospital, Sheffield, UK

\subsection{6/thoraxjnl-2016-209333.117}

Background Previous studies have demonstrated survival in systemic sclerosis ( $\mathrm{SSc}$ )-associated pulmonary arterial hypertension (SSc-PAH) to be worse than in idiopathic pulmonary arterial hypertension (IPAH). Possible explanations include age, differences in the underlying pulmonary vasculopathy and the ability of the right ventricle to compensate for the increased afterload. ${ }^{1}$ We investigated differences between these conditions by comparing demographic, haemodynamic and cardiac magnetic resonance imaging (MRI) characteristics and outcomes in a large cohort of incident, treatment-naive patients.

Methods 183 patients with IPAH and 192 patients with SSc-PAH were identified from departmental databases including the ASPIRE registry. ${ }^{2}$ Sub-group analysis in 83 patients who had undergone cardiac MRI within 14 days of right heart catheterisation was performed.

Results Median survival in IPAH was 7.8 years and in SSc-PAH was 3 years $(p<0.001)$. Patients with SSc-PAH were older with milder pulmonary haemodynamics but lower gas transfer $\left(\mathrm{DL}_{\mathrm{CO}}\right)$. Independent prognostic factors at multivariate Cox regression analysis were age, presence of systemic sclerosis, $\mathrm{DL}_{\mathrm{CO}}$, pulmonary artery saturation and stroke volume. For a given resistance (R), pulmonary arterial compliance (C) was reduced (lower RC) in SSc-PAH (Figure). The relationship between mean pulmonary arterial pressure (mPAP) and systolic pulmonary arterial pressure (sPAP) in IPAH was identical to that previously reported $(\mathrm{mPAP}=0.61 \mathrm{sPAP}+2 \mathrm{mmHg})$. The relationship in SSc-PAH was found to be: $\mathrm{mPAP}=0.58 \mathrm{sPAP}+2$ $\mathrm{mmHg}$ ( $\mathrm{p}$-value for difference with IPAH $=0.095)$. There was

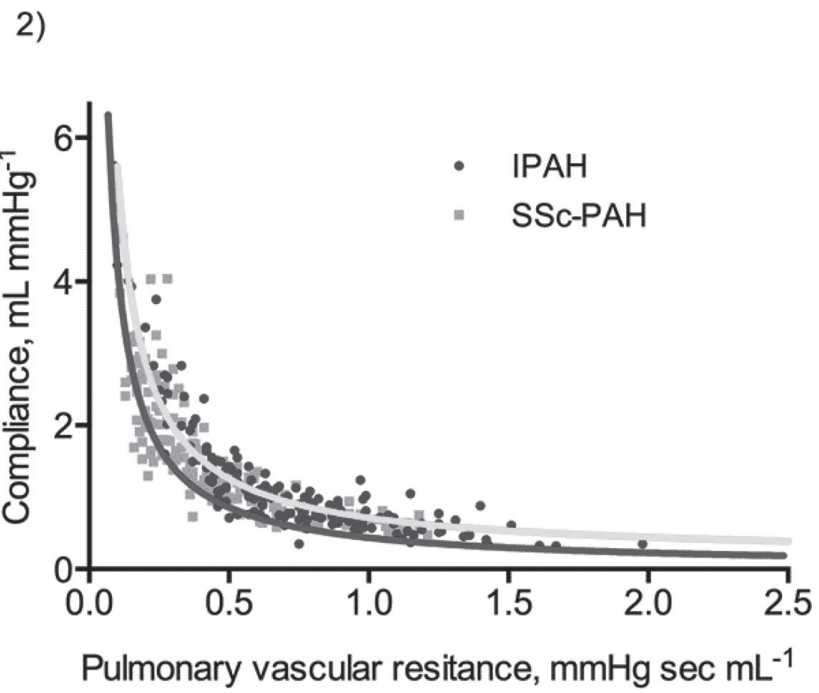

Abstract S111 Figure 1 